\title{
The influence of cohesive zone modeling on the stress field and energy release rates in a cracked elastic body
}

\author{
Pavana Prabhakar,* and Anthony M. Waas ${ }^{\dagger}$ \\ College of Engineering, University of Michigan, Ann Arbor, MI 48109-2140, USA
}

\begin{abstract}
The characteristic singular field at the tip of a crack in a linear elastic, homogeneous material was investigated by Williams, ${ }^{1}$ who studied the planar stress field in a traction free wedge with vertex angle $2 \alpha$. When $\alpha=\pi$, the traction free surfaces of the wedge fold into a "mathematically sharp" crack with vanishing tip radius, resulting in a stress field that varies with distance, $r$, from the crack tip as $r^{-\frac{1}{2}}$. In this paper, a traction free plane stress wedge with a discrete cohesive zone along the intended crack-path is studied. Of interest is the influence of the cohesive zone on the crack tip stress field. Further, the influence of the cohesive zone on the strain energy release rate is also investigated. Two example problems are studied; a double cantilever beam and an infinitely wide plate with a through-the-thickness crack. The objective of this paper is to examine the conditions under which a cohesive zone model can be used to represent an intended crack-path without unduly altering the original problem being investigated.
\end{abstract}

\section{Introduction}

Computational modeling of cracked bodies and layered structures with interfaces are of importance in the analysis of structures made of composite materials. In order to simulate the transition between a continuum and a non-continuum in an uninterrupted manner, the cohesive zone modeling (CZM) framework has gained popularity and is being incorporated into commercial finite element (FE) software packages (two examples are the commercial finite element packages, ABAQUS and ANSYS) that are used for stress analysis. In the CZM framework, originally introduced by Barenblatt, ${ }^{2}$ the material ahead of the intended crack path is replaced by elements that have a prescribed traction law as discussed in, for example, Gustafson and Waas, ${ }^{3}$ Song and Waas, ${ }^{4,5}$ Qui et al. ${ }^{6}$ Li et al. ${ }^{7}$ and Freed and Banks-Sills. ${ }^{8}$ This traction law is supposed to capture the physical attributes of the material or interface (for example, in case of adhesively bonded joints, the adhesive layer can be modeled as a CZ layer, see, Gustafson and Waas ${ }^{3}$ ), usually, through two parameters; a cohesive strength, $\sigma_{c}$ and a fracture toughness, $G_{c}$. Thus, when a CZM strategy is used, the original boundary value problem that contains the body $\left(B_{0}\right)$ to be analyzed is replaced by a new body $\left(B_{1}\right)$ that has a CZM contained within it. In the case of a cracked body, $\left(B_{1}\right)$, two issues are of concern: (a) To what extent are the features of the crack tip stress field that is originally contained in $\left(B_{0}\right)$ different than in $\left(B_{1}\right)$ ? (b)How is the strain energy release rate (SERR) corresponding to $\left(B_{0}\right)$ different from that which is computed in $\left(B_{1}\right)$ ? In other words, to what degree is the SERR of $\left(B_{0}\right)$ different from that which is computed in $\left(B_{1}\right)$ ? Some of these issues for elastic-plastic fracture have been addressed in recent work by Sun et al. ${ }^{9,10}$ In particular, these authors have considered two issues; (1) when CZM is used, the placement of CZM elements along the intended crack path can lead to an alteration of the stiffness of the original body that is to be studied, and, (2) Since the traction-separation laws used for traditional CZM modeling, which start with a vanishing traction at vanishing separation, are in conflict with the requirement of an intense stress field that was present in the original body $\left(B_{0}\right)$ that is being modeled, to what extent is the choice of traction law influencing computational results that are generated using CZM strategies?

\footnotetext{
* Graduate Student, Department of Aerospace Engineering.

${ }^{\dagger}$ Felix Pawlowski Collegiate Professor, Department of Aerospace Engineering and Department of Mechanical Engineering. Associate Fellow, AIAA.
} 
The objective of this paper is to examine these questions by analyzing a plane stress bi-material wedge with a CZM added along the interface of the two materials. The CZM is described by traction-separation laws for the opening (mode I) and sliding (mode II) modes. Both, homogenous (when the two wedge materials are the same) and bi-material wedges are considered and their response to crack initiation is studied for different values of CZM parameters. As examples, a double-cantilever beam and an infinitely wide plate with a through crack subjected to tensile forces are studied, both of which correspond to mode I fracture. The stress fields, SERR and stress intensity factors, (SIFs) are compared for specimens with and without the CZM to understand the influence of using a CZM in computational modeling of structures.

\section{Problem Formulation}

The original model of the plane stress wedge used in Williams ${ }^{1}$ to analyze the stresses at the crack tip in a plane stress problem, is shown in Fig. 1 . When $\alpha$ approaches $\pi$ in zone 1 , and $-\pi$ in zone 2 , the wedge can become a traction free, mathematically sharp crack. The Williams model is replaced by two symmetrical wedges (symmetric about the interface, ) with different homogeneous materials in each wedge. These wedges are separated by a CZM that is represented as a continuous bed of nonlinear 1D cohesive elements, which have a prescribed traction-separation relation, as shown in Fig. 2.

The case of a free-free wedge is considered here. That is, traction-free surfaces are considered, so that for appropriate wedge angles, a crack that has a CZM ahead of it can be simulated.

The governing equilibrium field equations and associated boundary and matching conditions are derived using the principle of virtual work. The derived equations are solved for the case of linear elastic materials, with a CZM that has a triangular traction-separation law, with an initial linear stiffness. A brief overview of the derivation is given below.

The incremental strain energy, $\delta U$, of the two wedges, the incremental work of tractions in the CZM, and the external incremental boundary work, $\delta W_{E}$, for the entire body, with zero body forces, is as given below.

$$
\delta U=\int_{\Omega_{1}} \underline{\sigma}: \underline{\delta \epsilon} d v_{1}+\int_{\Omega_{2}} \underline{\sigma}: \underline{\delta \epsilon} d v_{2}+\int_{S_{i}^{1}} T_{n}^{C Z M} \Delta u d s+\int_{S_{i}^{1}} T_{s}^{C Z M} \Delta v d s
$$

where, $\Delta u$ is $\delta\left(u_{1}-u_{2}\right), \Delta v$ is $\delta\left(v_{1}-v_{2}\right), T_{n}^{C Z M}$ is the normal traction in the cohesive zone and $T_{s}^{C Z M}$ is the shear traction in the cohesive zone.

$$
\delta W_{E}=\int_{S_{1}^{o}} T_{1}^{1 o} \delta v_{1} d s+\int_{S_{1}^{o}} T_{2}^{1 o} \delta u_{1} d s-\int_{S_{2}^{o}} T_{1}^{2 o} \delta v_{2} d s-\int_{S_{2}^{o}} T_{2}^{2 o} \delta u_{2} d s
$$

$\delta W_{E}$ is the external incremental work done, by the tractions $\left\{T_{1}^{1 o}, T_{2}^{1 o}\right\}$ on the outer surface of zone 1 and the tractions $\left\{T_{1}^{2 o}, T_{2}^{2 o}\right\}$ on the outer surface of zone 2 , respectively. $\delta u_{1}$ and $\delta u_{2}$ are the kinematically compatible incremental displacements in the two zones in the $\theta$ direction, and $\delta v_{1}$ and $\delta v_{2}$ are the kinematically compatible incremental displacements in the two zones in the $r$ direction. The strain energy and the external work done for linear elastic materials take the following form,

$$
U=\int_{\Omega_{1}} \underline{\sigma}: \underline{\epsilon} d v_{1}+\int_{\Omega_{2}} \underline{\sigma}: \underline{\epsilon} d v_{2}+\int_{S_{i}^{1}} \frac{1}{2} k_{2}\left[u_{1}-u_{2}\right]^{2} d s+\int_{S_{i}^{1}} \frac{1}{2} k_{1}\left[v_{1}-v_{2}\right]^{2} d s
$$

External work :

$$
W_{E}=\int_{S_{1}^{o}} T_{1}^{1 o} v_{1} d s+\int_{S_{1}^{o}} T_{2}^{1 o} u_{1} d s-\int_{S_{2}^{o}} T_{1}^{2 o} v_{2} d s-\int_{S_{2}^{o}} T_{2}^{2 o} u_{2} d s
$$

Using the principle of virtual work, we get the equations of equilibrium and the corresponding boundary and matching conditions for each of the two wedges. The equations of equilibrium are,

$$
\begin{gathered}
\frac{1}{r} \frac{\partial \sigma_{\theta}}{\partial \theta}+\frac{\partial \tau_{r \theta}}{\partial r}+\frac{2 \tau_{r \theta}}{r}=0 \quad \text { in } \quad \Omega_{1} \\
\frac{\partial \sigma_{r}}{\partial r}+\frac{\left(\sigma_{r}-\sigma_{\theta}\right)}{r}+\frac{1}{r} \frac{\partial \tau_{r \theta}}{\partial \theta}=0 \quad \text { in } \quad \Omega_{1} \\
\frac{1}{r} \frac{\partial \sigma_{\theta}}{\partial \theta}+\frac{\partial \tau_{r \theta}}{\partial r}+\frac{2 \tau_{r \theta}}{r}=0 \quad \text { in } \quad \Omega_{2}
\end{gathered}
$$




$$
\frac{\partial \sigma_{r}}{\partial r}+\frac{\left(\sigma_{r}-\sigma_{\theta}\right)}{r}+\frac{1}{r} \frac{\partial \tau_{r \theta}}{\partial \theta}=0 \quad \text { in } \quad \Omega_{2}
$$

and the corresponding 4 boundary and 4 matching conditions are,

$$
\begin{gathered}
\sigma_{\theta}=T_{2}^{1 o} \text { on } \quad \mathrm{S}_{1}^{\mathrm{o}} \\
\tau_{r \theta}=T_{1}^{1 o} \text { on } \quad \mathrm{S}_{1}^{\mathrm{o}} \\
\sigma_{\theta}=T_{2}^{2 o} \text { on } \quad \mathrm{S}_{2}^{\mathrm{o}} \\
\tau_{r \theta}=T_{1}^{2 o} \text { on } \quad \mathrm{S}_{2}^{\mathrm{o}} \\
\int_{S_{i}} \sigma_{\theta} d s=\int_{S_{i}} k_{2}\left[u_{1}-u_{2}\right] d s \text { on } \mathrm{S}_{\mathrm{i}}^{1} \\
\int_{S_{i}} \tau_{r \theta} d s=\int_{S_{i}} k_{1}\left[v_{1}-v_{2}\right] d s \text { on } \mathrm{S}_{\mathrm{i}}^{1} \\
\left.\sigma_{\theta}\right|_{S_{i}^{1}}=\left.\sigma_{\theta}\right|_{S_{i}^{2}} \\
\left.\tau_{r \theta}\right|_{S_{i}^{1}}=\left.\tau_{r \theta}\right|_{S_{i}^{2}}
\end{gathered}
$$

These equations are first solved for linear elastic materials. Furthermore, it is assumed that the initial stiffness of the CZM is linear, conforming to a triangular traction-separation law. By choosing the following definitions for the stresses, $\sigma_{r}=\frac{1}{r} \frac{\partial \chi}{\partial r}+\frac{1}{r^{2}} \frac{\partial^{2} \chi}{\partial \theta^{2}}, \sigma_{\theta}=\frac{\partial^{2} \chi}{\partial r^{2}}$ and $\tau_{r \theta}=-\frac{\partial}{\partial r}\left(\frac{\partial \chi}{r \partial \theta}\right)$, along with the equilibrium equations satisfied identically, we get the classical bi-harmonic equation by enforcing the compatibility of strains as,

$$
\nabla^{4} \chi(r, \theta)=0
$$

to be satisfied in each of the two zones. Therefore, guided by the approach in Williams, ${ }^{1}$ a solution to the above equation is assumed to be:

$$
\begin{aligned}
\chi(r, \theta, \lambda) & =r^{\lambda+1}\left[a_{1} \sin (\lambda+1) \theta+b_{1} \cos (\lambda+1) \theta+c_{1} \sin (\lambda-1) \theta+d_{1} \cos (\lambda-1) \theta\right] \text { in } \Omega_{1} \\
& =r^{\lambda+1} F_{1}(\theta ; \lambda)
\end{aligned}
$$

and

$$
\begin{aligned}
\chi(r, \theta, \lambda) & =r^{\lambda+1}\left[a_{2} \sin (\lambda+1) \theta+b_{2} \cos (\lambda+1) \theta+c_{2} \sin (\lambda-1) \theta+d_{2} \cos (\lambda-1) \theta\right] \text { in } \Omega_{2} \\
& =r^{\lambda+1} F_{2}(\theta ; \lambda)
\end{aligned}
$$

The 4 boundary and 4 matching conditions for the entire wedge with the cohesive zone with an including angle equal to $2 \pi$, in terms of $r, \theta$ coordinates are as follows :

$$
\begin{gathered}
\sigma_{\theta 1}=0 \text { at } \theta=\pi \\
\tau_{r \theta 1}=0 \text { at } \theta=\pi \\
\sigma_{\theta 2}=0 \text { at } \theta=-\pi \\
\tau_{r \theta 2}=0 \text { at } \theta=-\pi \\
\sigma_{\theta 1}=\sigma_{\theta 2} \text { at } \theta=0 \\
\tau_{\theta 1}=\tau_{\theta 2} \text { at } \theta=0
\end{gathered}
$$

For the last two conditions, we introduce a characteristic length denoted by ' $L$ '. This length can be chosen based on the CZM properties, for example, and is defined as, $L=\frac{E G_{I C}}{\sigma_{c}{ }^{2}}$. Alternatively, this length can also be defined as $L=\frac{1}{2 \pi}\left(\frac{K_{I}}{\sigma_{Y}}\right)^{2}$ in case of elasto - plastic material.A circular region of diameter, $L$, surrounding the crack tip, is referred to as the zone of dominance and it is expected that this region engulfs any dominant singularities in the problem. For the purpose of computation presented here, $L$ is estimated as, 
$L=66 \mathrm{~mm}$, using $L=\frac{E G_{I C}}{\sigma_{c}{ }^{2}}$, where, $E=3.3 G P a, G_{I C}=500 \mathrm{~N} / \mathrm{m}$ and $\sigma_{c}=5 \mathrm{MPa}$. The 7 th and the 8th boundary conditions are now cast in integral form, to be valid within the zone of dominance as,

$$
\int_{0}^{L} \sigma_{\theta 1} d r=\int_{0}^{L} T_{n}^{C Z M} d r
$$

and,

$$
\int_{0}^{L} \tau_{r \theta 1} d r=\int_{0}^{L} T_{s}^{C Z M} d r
$$

Applying the above 8 boundary conditions, 8 homogenous equations in 8 unknowns $\left(a_{1}, b_{1}, c_{1}, d_{1}, a_{2}, b_{2}, c_{2}, d_{2}\right)$ are obtained, and these equations are cast as a system of linear algebraic equations,

$$
\underline{A} \vec{X}=\overrightarrow{0}
$$

where, $\vec{X}$ is a vector of the unknowns. For non-trivial solution, the determinant of $\underline{A}$ must vanish, which results in an equation for $\lambda$, with other material and CZM properties entering the equation. The solution provides $\lambda$ for various combinations of material properties and CZM parameters. To start with, in the next section, the homogeneous case is considered first (with same material properties for the two bodies attached through a CZM).

\section{A. Traction - Separation Law}

The traction separation law used is a triangular law, and is adopted from Xie and Waas ${ }^{11}$ with a maximum traction $\sigma_{c}$ and a fracture toughness $G_{I C}$ as shown in Fig. 3.

With known values of $\sigma_{c}, G_{I C}$ and $\delta_{1}$, the corresponding values of initial stiffness of the cohesive zone is determined. That is, from the triangle in Fig. 3, the value of ' $k$ ' is determined as follows: The area of the triangle is $G_{I C}$ and is given by,

$$
G_{I C}=\frac{1}{2} \sigma_{c} \delta^{\star}
$$

The slope ' $k$ ' is given by,

$$
k=\frac{\sigma_{c}}{\delta_{1}}
$$

Keeping the area of the triangle constant, i.e. $G_{I C}$ constant, $\delta_{1}$ is varied to obtain different values of initial slope ' $k$ ' as shown in 4 . That is, when $\delta_{1}=0$, the maximum stiffness $k_{\max }=\infty$, and when $\delta_{1}=\delta^{\star}$, the minimum stiffness $k_{\text {min }}=\frac{\sigma_{c}}{\delta^{\star}}$. This implies that the values of stiffness can be varied between $k_{\min }$ and $k_{\max }$, $\frac{\sigma_{c}}{\delta^{\star}}<k<\infty$.

\section{Homogeneous Case}

The homogenous case is considered first. It is assumed that both zones 1 and 2 in the body have the same material properties and are defined by the same Lamé constants, i.e. $\mu_{1}=\mu_{2}$. Also, the initial stiffness of the cohesive zone is assumed to be equal in the opening and sliding modes. As shown in the previous section, with the known values of $G_{I C}$ and $\sigma_{c}$, we have a range of values for $k$. The range of stiffness varies from 10 to $\infty$ ( practically, a very high value $\approx 10^{5}$ ). Solving, for $\lambda$ in the determinant of $\underline{A}$, for different values of $k$ and fixing the characteristic length to $66 \mathrm{~mm}$, we plot $\lambda$ against $k$ as shown in Fig. 5 . We notice here that, as $k$ varies from 10 to $10^{5}$ on a logarithmic scale, $\lambda$ varies between 1 and 0.5 . This matches with the results given in Williams. ${ }^{12}$ That is, for the wedge shown in Fig. 1, when the vertex angle between the faces of the wedge is $2 \pi, \lambda$ is 0.5 for a free-free homogeneous wedge without a CZM. For example, the $\sigma_{\theta 1}$ along the $\mathrm{CZM} /$ crack path is given by,

$$
\sigma_{\theta 1}=r^{\lambda-1}\left[\lambda(\lambda+1) F_{1}(\theta=0)\right]
$$

and, $\sigma_{\theta 1}$ for the case with the CZM is given by,

$$
\sigma_{\theta 1}=r^{\lambda-1} \lambda(\lambda+1)(b 1+d 1)
$$

where, $b 1$ and $d 1$ are constants which depend on the material properties of the wedges. A plot of $\sigma_{\theta}$ against length along the CZM path from CZM model and the Williams solution ${ }^{1}$ for different values of $k$, are plotted 
in Fig. 6. Here, as the stiffness of the cohesive zone is increased tending to $\infty$, the value of $\lambda$ tends towards 0.5 , which gives a $r^{-\frac{1}{2}}$ singularity in stress field in the vicinity of the crack/wedge tip. However, for other values of $k$, it is seen that the crack tip singularity is different from -0.5 and is, in general, weaker. Consequently, it is expected that both the stress intensity factor and SERR will be affected, since these depend on the strength of the singularity.

Also, the variation of displacement jump normal to the crack length and along the interface between the wedges is examined for different values of initial CZM stiffness. The plot of $\Delta u_{\theta}$ along $r$ for different values of $k$ at $\theta=0$ obtained from CZM model as well as Williams solution ${ }^{1}$ is shown in Fig. 7. We notice that as the initial stiffness of cohesive zone is increased, the displacements get closer to the Williams solution. From the above it can be inferred that in order to obtain the same behavior in stresses and displacements in a model with CZM elements, it is necessary that the initial stiffness of cohesive zone is very high, and we characterize this initial stiffness in terms of a non-dimensional parameter, $\rho$, later in the paper.

\section{Bi-Material Case}

In this section, the two wedges separated by the CZM are considered to possess different material properties. This is controlled by taking $\mu_{1} \neq \mu_{2}$. We introduce a parameter, $\alpha$, which is one of the Dundur's Parameters adopted from Suo, ${ }^{13}$ and is defined as,

$$
\alpha=\frac{\Gamma\left(\phi_{2}+1\right)-\left(\phi_{1}+1\right)}{\Gamma\left(\phi_{2}+1\right)+\left(\phi_{1}+1\right)}
$$

where, $\Gamma=\frac{\mu_{1}}{\mu_{2}}, \phi_{i}=\frac{3-\nu_{i}}{1+\nu_{i}}$ and $\mathrm{i}=1,2$ corresponding to each of the zones 1 and 2 . By simple manipulations we get $\Gamma$ as follows,

$$
\Gamma=\frac{\mu_{1}}{\mu_{2}}=\frac{(1+\alpha)\left(\phi_{1}+1\right)}{(1-\alpha)\left(\phi_{2}+1\right)}
$$

Therefore, substituting for $\mu_{1}$ in the determinant, we get an expression in terms of $\lambda, \mu_{2}, \alpha, \nu_{1}, \nu_{2}, k_{1}, k_{2}$ and $L$. For a range of values of $\alpha$, and for chosen values of $\nu_{1}$ and $\nu_{2}$, and for different values of $k\left(k_{1}=k_{2}\right)$, we find corresponding values of $\lambda$. A surface plot of $\lambda$ vs $k$, for varying $\alpha$ is shown in Fig. 8 . Here, the value of $\alpha$ describes the bi-material property of the wedge, i.e., for $\alpha=0$, the wedge becomes homogenous. So, it can be seen in Fig. 8 that for a range of values of $\alpha$, the value of $\lambda$ gets closer to 0.5 as the stiffness of the cohesive zone is excessively increased. This implies that even for a bi-material wedge, a similar trend is observed as in the case of a homogenous wedge, with the strength of the singularity dependent on the initial CZM stiffness, $k$.

\section{Example Problems}

In this section, a double cantilever beam configuration and a thin plate with a central crack are analyzed to illustrate the findings presented earlier.

\section{A. Double cantilever beam}

The DCB with CZM is shown in Fig. 9. Forces "P" are applied to either end of the DCB arms, and are pulled apart causing bending of DCB arms (flanges). The stress intensity factor (SIF) of the model is calculated as a function of the stiffness of the cohesive zone ${ }^{\mathrm{a}}$. To compare the models with and without cohesive zones, we calculate the percentage error in the values of SIF (denoted as, Err), as follows :

The analytical model of the DCB is as shown in Fig. 10. Strain energies and displacement fields of the two parts 1 and 2 are given by,

$$
U_{1}=\int_{0}^{a} \frac{1}{2} E I\left(w_{1}^{\prime \prime}\right)^{2} d x
$$

and,

$$
U_{2}=\int_{a}^{L} \frac{1}{2} k\left(w_{2}\right)^{2} d x+\int_{a}^{L} \frac{1}{2} E I\left(w_{2}^{\prime \prime}\right)^{2} d x
$$

\footnotetext{
a Note that for an isotropic material under mode-I fracture, SIF is related to SERR by, $G_{I}=\frac{\left(1-\nu^{2}\right)}{E} K_{I}^{2}$ for plane strain and $G_{I}=\frac{K_{I}^{2}}{E}$ for plane stress
} 
where,

$$
\begin{gathered}
w_{1}=\frac{1}{6} \frac{P}{E I} x^{3}+-\frac{1}{2} \frac{P\left(a^{2} \lambda^{2}+2 a \lambda+1\right)}{E I \lambda^{2}} x+\frac{1}{6} \frac{P\left(2 a^{3} \lambda^{3}+6 a^{2} \lambda^{2}+6 \lambda a+3\right)}{E I \lambda^{3}} \\
w_{2}=e^{(-\lambda x)}\left[\frac{1}{2} \frac{(\cos (\lambda a)+\sin (\lambda a))\left(\sin (\lambda a)^{2}-1-\lambda a+2 \lambda a \sin (\lambda a)^{2}+\cos (\lambda a) \sin (\lambda a)\right) P}{\left(2 \sin (\lambda a)^{2}-1\right) \lambda^{3} e^{(-\lambda a)} E I} \cos (\lambda x)\right. \\
\left.+\frac{1}{2} \frac{P(-\cos (\lambda a)+\sin (\lambda a))\left(\cos (\lambda a) \sin (\lambda a)-\lambda a+2 \lambda a \sin (\lambda a)^{2}+\sin (\lambda a)^{2}\right)}{\left(2 \sin (\lambda a)^{2}-1\right) \lambda^{3} e^{(-\lambda a)} E I} \sin (\lambda x)\right]
\end{gathered}
$$

where,

$$
\lambda=\left(\frac{k}{4 E I}\right)^{\frac{1}{4}}
$$

Then, the total strain energy is given by,

$$
U=\int_{0}^{a} \frac{1}{2} E I\left(w_{1}^{\prime \prime}\right)^{2} d x+\int_{a}^{a+y} \frac{1}{2} k\left(w_{2}\right)^{2} d x+\int_{a}^{a+y} \frac{1}{2} E I\left(w_{2}^{\prime \prime}\right)^{2} d x
$$

Let,

$$
X=\frac{x}{a}, Y=\frac{y}{a}, W_{1}=\frac{w_{1}}{h}, W_{2}=\frac{w_{2}}{h}, k=\frac{\beta E I}{t a^{4}}, \bar{P}=\frac{P a^{3}}{h E I}
$$

The corresponding non-dimensional strain energy is,

$$
\bar{U}=\frac{U}{2} \frac{a^{3}}{E I h^{2}}
$$

The stress intensity factor (SIF) is defined as, ${ }^{14}$

$$
K_{I}^{2}=E \frac{1}{t} \frac{\partial U}{\partial a}
$$

Therefore, the non-dimensional SIF is,

$$
K_{I_{n d}}=K_{I} \sqrt{\left(\frac{t a^{4}}{h^{2} E^{2} I}\right)}
$$

Now, considering the model without the cohesive zone, the equation for the SIF is given by,

$$
K_{I_{a}}=P a \sqrt{\frac{1}{I t}}
$$

and, the non-dimensional SIF is,

$$
K_{I_{a-n d}}=\bar{P}
$$

The material chosen for the beam has Young's modulus $E=70 \mathrm{GPa}$. The dimensions of the beam are as follows: $a=0.5 m, h=0.05 m, t=0.05 \mathrm{~m}$. The value of the non-dimensional force, $\bar{P}=100$. The value of the non-dimensional stiffness ' $\beta$ ' for the cohesive zone is varied from 10 to $10^{9}$. The percentage error in the values of $K_{I_{n d}}$ and $K_{I_{a-n d}}$ is calculated, and the error is plotted against the entire range of stiffness of the cohesive zone in Fig. 11. We notice that the error tends to zero as the initial stiffness of the cohesive zones tend to $\infty$. Moreover, in the model with CZM, the contribution of the springs to the SIF is studied. A plot of SIF contribution of the springs is plotted against the total SIF of the model as shown in Fig. 12. It is noticed that, as the stiffness of the springs is increased, its contribution to SIF reduces as expected. The tip displacement is also plotted against tip load for a range of spring stiffness, and is compared to the DCB with no springs in Fig. 13, illustrating how the initial stiffness of the CZM alters the load-deflection response, which is a measure of the structural stiffness. 


\section{B. Infinitely wide thin plate with a central crack}

The infinitely wide plate with a center crack is shown in Fig. 14. We again calculate the percentage error in the values of SIF for a model with and without a cohesive zone as shown below.

Using the principle of superposition as shown by Zok, ${ }^{15}$ the SIF of the plate is determined by adding the SIF of two different plates as shown in Fig. 15. SIF for part A is given in Anderson ${ }^{14}$ and, is as follows:

$$
K_{I_{A}}=\sigma_{o} \sqrt{\pi a}
$$

For part B, the strain energy is given by the following expression:

$$
U=-\frac{K_{I_{A}}^{2} B}{\pi k}\left[\log \left(\frac{a}{a-L}\right)\right]
$$

Thus, the SIF for part B is,

$$
\begin{aligned}
\frac{K_{I_{B}}^{2}}{E}=\frac{1}{B} \frac{\partial U}{\partial a} & =-\frac{K_{I_{A}}^{2}}{\pi k}\left[\frac{1}{a}-\frac{1}{a-L}\right] \\
\frac{K_{I_{B}}^{2}}{E} & =\frac{\sigma_{o}^{2} L}{k(a-L)}
\end{aligned}
$$

Therefore,

$$
K_{I_{B}}=\sigma_{o} \sqrt{\frac{E L}{k(a-L)}}
$$

The total SIF of the plate is,

$$
\begin{gathered}
K_{I}=K_{I_{A}}+K_{I_{B}} \\
K_{I}=\sigma_{o} \sqrt{\pi a}+\sigma_{o} \sqrt{\frac{E L}{k(a-L)}}
\end{gathered}
$$

Thus, the error in the SIF is $\frac{K_{I_{B}}}{K_{I_{A}}}$ and is given by,

$$
\text { error }=\sqrt{\frac{E L}{\pi k a(a-L)}}
$$

The percentage error is plotted against the entire range of stiffness of the cohesive zone in Fig. 16. We observe the same pattern of response in the error as in the case of the DCB.

These results show that the insertion of a CZM can (and does) alter the deformation response of a body in a computational modeling CZM framework. On the other hand, methods which model the body as a continuum but transition seamlessly to a CZM strategy later in the loading history - such as in the VMCM method, ${ }^{16}$ does not lead to an alteration in the responses of the body being modeled. From the P- $\Delta$ curve for the DCB shown in Fig. 13, it is evident that the initial stiffness of the cohesive zone affects the load deflection behavior. It is noticed that as the CZM stiffness becomes greater than the elastic modulus of the DCB material, the P - $\Delta$ curve converges to the one without CZM. Again, we quantify the necessary increase in the initial CZM stiffness in terms of $\rho$ as detailed later.

\section{Effect of the initial CZM stiffness, $k$}

The analysis of the wedges with CZM inserted is conducted for three different characteristic lengths, $(\mathrm{L}=66 \mathrm{~mm}, 33 \mathrm{~mm}, 3.3 \mathrm{~mm})$. The stress $\sigma_{\theta}$ is determined for all the three lengths for two different cases : (1) Varying the critical traction of the cohesive law $\left(\sigma_{c}\right)$ with constant elastic modulus, $E$, of the wedges, (2) Varying elastic modulus of the wedges with constant critical traction of the cohesive law, $\left(\sigma_{c}\right)$. The stresses computed are compared with the stress $\sigma_{\theta}$ from the Williams solution (without a CZM) at a distance $r=2 \mathrm{~mm}$ from the crack tip at $\theta=0$. In order to get these values to match within a desired percentage of 0.3 percent, the initial stiffness of the cohesive zone required is determined for each case. Two

new non-dimensional parameters are constructed as follows : $\rho=\frac{k G_{I C}}{\sigma_{c}^{2}}$ and $E / \sigma_{c}$, which will aid towards determining a measure for $k$. 
Properties used in case 1 are $G_{I C}=0.5 \frac{\mathrm{N}}{\mathrm{mm}}$ and $E=3.3 \mathrm{GPa}$. The $\sigma_{c}$ corresponding to the three different characteristic lengths, $L$, are $5,7.07$ and $22.36 \mathrm{MPa}$. The initial stiffnesses are determined to be $5 e 3,1 e 4$ and $1 e 5 \frac{\mathrm{N}}{\mathrm{mm}^{3}}$, respectively. Similarly, properties used in case 2 are $G_{I C}=0.5 \frac{\mathrm{N}}{\mathrm{mm}}$ and $\sigma_{c}=10 \mathrm{MPa}$. The $E$ corresponding to the three different lengths $(L)$ are 13.2,6.6, 0.660 GPa, respectively. The initial stiffness is found to be $k=2 e 4 \frac{N}{m^{3}}$ for all the three lengths.

For both cases, the non-dimensional parameters mentioned above are determined and plotted in Fig. 17. It is observed that the non - dimensional parameter $\rho$, is almost insensitive to $E / \sigma_{c}$, and is equal to 100. With this at hand, one can choose the value of $k$ for pre-determined values of $G_{I C}$ and $\sigma_{c}$ in such a way that $\rho$ is around 100, which would give stresses within the desired percentage when compared to the Williams solution.

As an example, the cohesive laws for $G_{I C}=0.5 \frac{\mathrm{N}}{\mathrm{mm}}$ and $E=3.3 \mathrm{GPa}$ and varying $\sigma_{c}$ values of 5, 7.07 and $22.36 \mathrm{MPa}$, and for values of $k$ determined from the analysis are plotted in Fig. 18. This shows that as long as $\rho$ is about 100 or greater, the local stress-field in the body outside the CZM region is computed to a sufficient level of accuracy.

\section{Concluding Remarks}

A stress free bi-material wedge with a vertex angle of $2 \pi$ has been considered to evaluate the effects of cohesive zone modeling in computational mechanics. The behavior of the stress field in the vicinity of a crack tip is studied by specializing the wedge angle to appropriate values. A homogenous case is considered where according to Fig. 6 , the stresses display $\frac{1}{\sqrt{r}}$ behavior for $\lambda=0.5$, in the absence of a cohesive zone. When a cohesive zone is inserted, the stress field, characterized in terms of the strength of the singularity is found to vary continuously with the initial cohesive zone stiffness, approaching $\frac{1}{\sqrt{r}}$ behavior only when the initial stiffness of the cohesive zone is very high. A non-dimensional parameter $\rho$ is introduced to characterize the required CZM initial stiffness. Further, a comparison of stresses and displacements from the Williams solution, ${ }^{1}$ and the solution obtained from a CZM approach for homogeneous material are presented in Fig. 6 and Fig. 7. It is observed that the variation of stress ' $\sigma_{\theta}$ ' and displacement ' $u_{\theta}$ ' at the interface is similar in both analysis only at very high initial stiffness of the cohesive zone, resulting in $\rho \geq 100$.

Along with the stress equivalence, results have been presented that describe the effects of the CZM on the stress intensity factor and the strain energy release rate through two example problems. In both the cases it is observed that percentage error in stress intensity factor reduces to be negligible only when the initial stiffness of the cohesive zone is very high.

Throughout the paper, it has been shown that in order to reproduce the same behavior of stresses, displacements and strain energy release rates in a model with cohesive zone when compared to a model without cohesive zones is possible, only when the initial stiffness of the cohesive zone is very high.

A non-dimensional parameter $\rho$ is established, which will serve as a guide in determining the initial stiffness of the cohesive law corresponding to pre-determined values of $G_{I C}$ and $\sigma_{c}$.

\section{References}

${ }^{1}$ Williams, M., "The stresses around a fault or crack in dissimilar media," Bulletin of the Seismological Society of America, Vol. 49, April 1959, pp. 199-204.

${ }^{2}$ Barenblatt, G., "The formation of equilibrium cracks during brittle fracture. General ideas and hypothesis. Axially symmetric cracks," J. Appl. Math. Mech., 1959.

${ }^{3}$ Gustafson, P. and Waas, A., "The influence of adhesive constitutive parameters in cohesive zone finite element models of adhesively bonded joints," International Journal of Solids and Structures, Vol. 46, No. 10, 2009, pp. $2201-2215$.

${ }^{4}$ Song, S. and Waas, A., "Mode-I Failure of Laminated Polymeric Composites," Engineering Fracture Mechanics, Vol. 49, No. 1, 1994, pp. 1727.

${ }^{5}$ Song, S. and Waas, A., "An Energy- based Mechanical Model for Mixed-mode Failure of Laminated Composites," AIAA J, Vol. 33, No. 4, 1995, pp. 73945.

${ }^{6}$ Qiu, Y., Crisfield, M., and Alfano, G., "An interface element formulation for the simulation of delamination with buckling," Engineering Fracture Mechanics, Vol. 68, No. 16, 2001, pp. 1755-1776.

${ }^{7} \mathrm{Li}, \mathrm{H}$. and Chandra, N., "Analysis of crack growth and crack-tip plasticity in ductile materials using cohesive zone models," Int J Plast, Vol. 19, No. 6, 2003, pp. 84982.

${ }^{8}$ Freed, Y. and Banks-Sills, L., "A new cohesive zone model for mixed mode interface fracture in bimaterials," Engineering Fracture Mechanics, Vol. 75, 2008, pp. 45834593.

${ }^{9}$ Jin, Z.-H. and Sun, C., "Cohesive Zone Modeling of Interface Fracture in Elastic Bi-Materials," Engineering Fracture Mechanics, Vol. 72, 2005. 
${ }^{10}$ Jin, Z.-H. and Sun, C., "A Comparison of Cohesive Zone Modeling and Classical Fracture Mechanics Based on Near Tip Stress Field," Int. J. Solids and Structures, Vol. 43, 2006.

${ }^{11}$ Xie, D. and Waas, A., "Discrete cohesive zone model for mixed-mode fracture using finite element analysis," Engineering Fracture Mechanics, Vol. 73, September 2006, pp. 1783-1796.

${ }^{12}$ Williams, M., "Stress singularities resulting from various boundary conditions in angular corners of plates in extension," American Society of Mechanical Engineers, February 1952.

${ }^{13}$ Suo, Z., "Singularities interacting with interface and cracks," Int. J. Solids Structures, Vol. 25, 1989, pp. $1133-1142$.

${ }^{14}$ Anderson, T., Fracture Mechanics : fundamentals and applications, CRC Press LLC, 1995.

${ }^{15}$ Zok, F. and Hom, C., "Large scale bridging in brittle matrix composites," Acta metall. mater., Vol. 38, March 1990, pp. 1895-1904.

${ }^{16}$ Rudraraju, S., Salvi, A., Garikipati, K., and Waas, A., "In-Plane fracture of laminated fiber reinforced composites with varying fracture resistance: experimental observations and numerical crack propagation simulations," International Journal of Solids and Structures, Vol. 47, No. 7, 2010. 


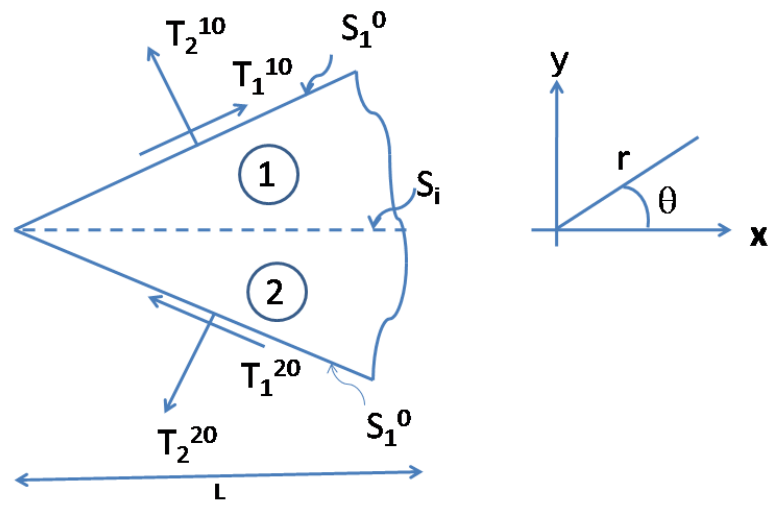

Figure 1. Bi-Material Wedge Subjected to Traction Boundary Conditions

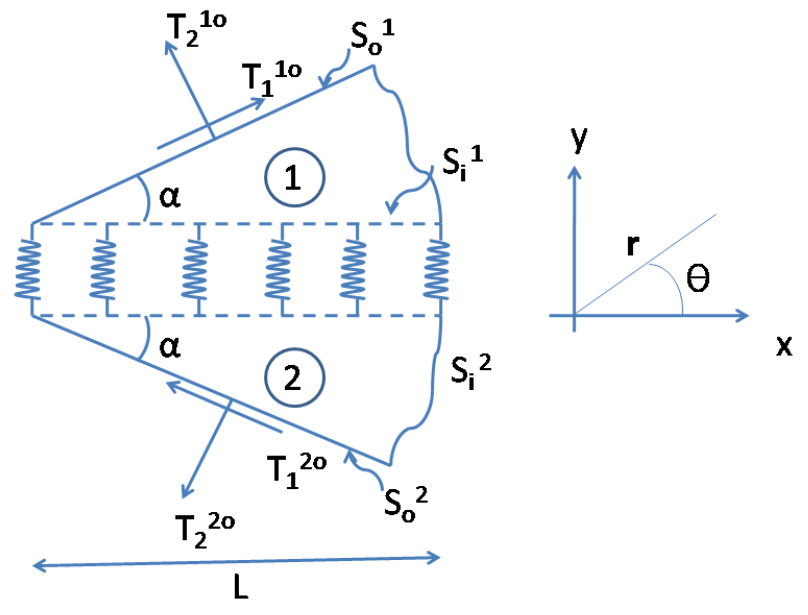

Figure 2. Bi-Material Wedge With Cohesive Zone Subjected to Traction Boundary Conditions 


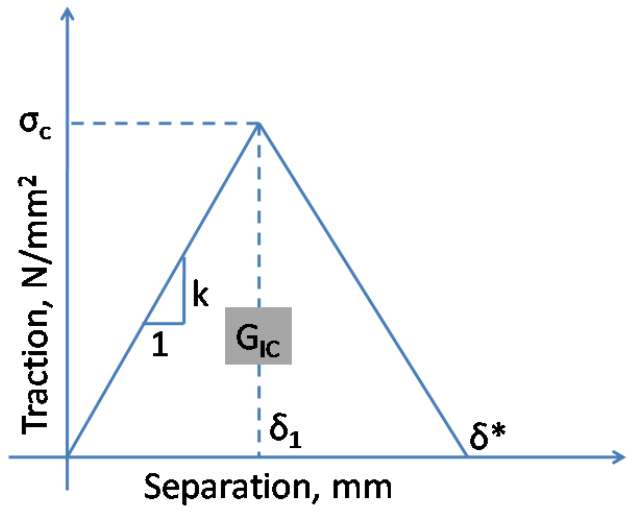

Figure 3. Traction - Separation Law for the Cohesive Zone

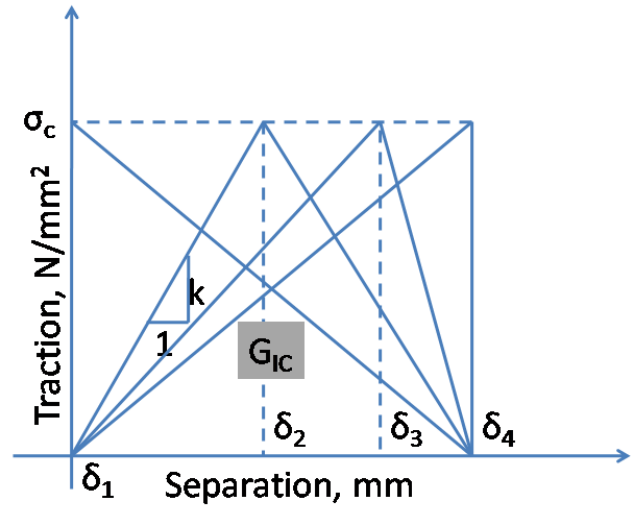

Figure 4. Traction - Separation Law for the Cohesive Zone with Fixed Fracture Toughness and Varying Stiffness 


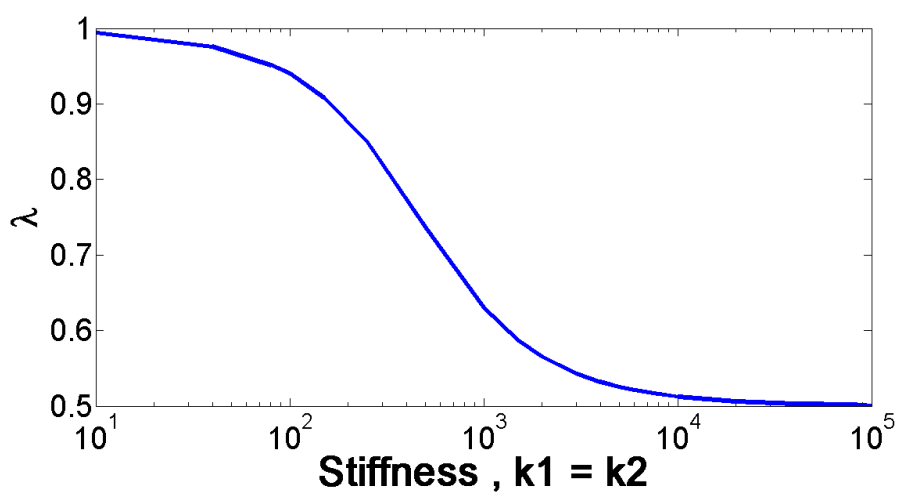

Figure 5. Plot of $\lambda$ vs Stiffness of the Cohesive Zone for Homogenous Case

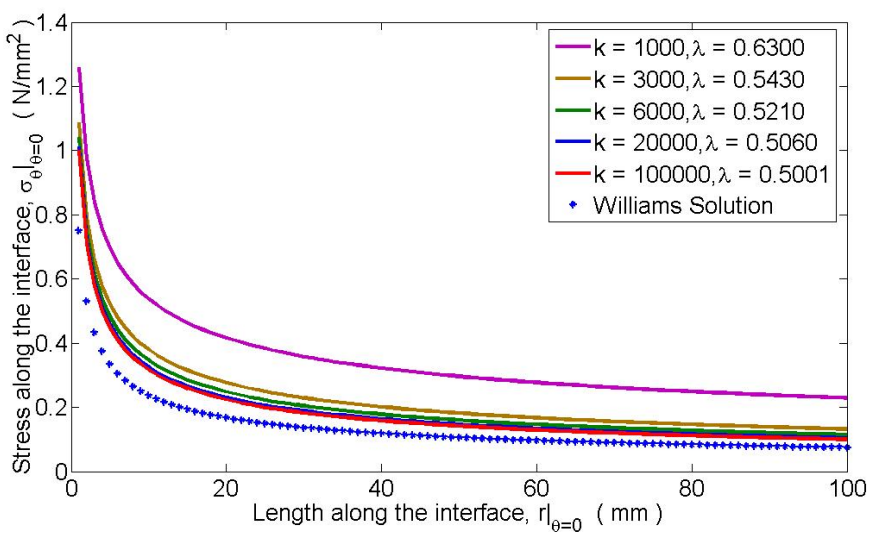

Figure 6. Plot of $\sigma_{\theta}$ vs Length Along Crack Path for Different Values of $k$

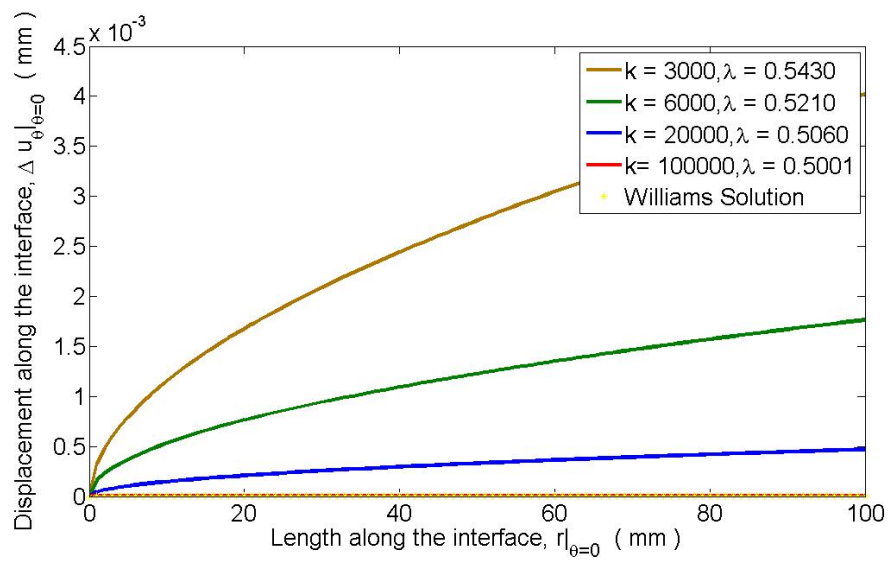

Figure 7. Plot of $u_{\theta}$ vs Length Along Crack Path for Different Values of $k$ 


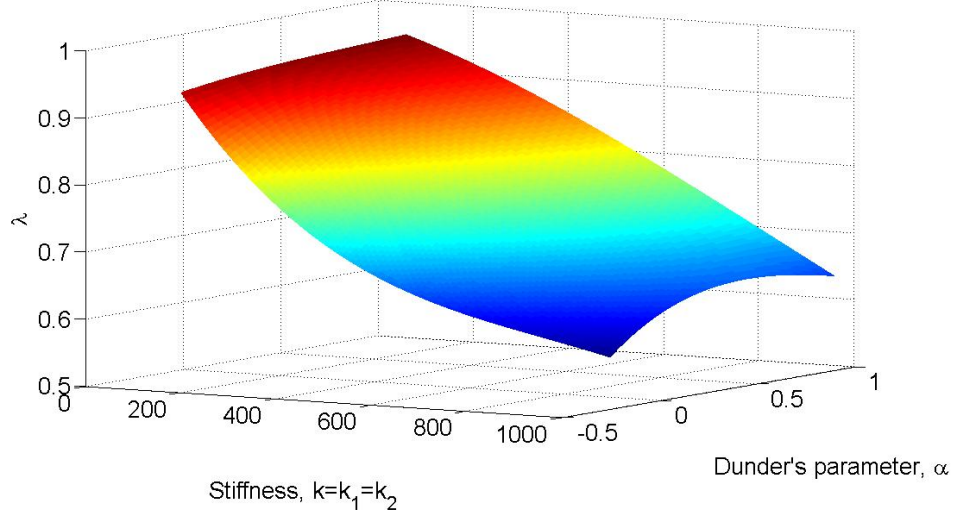

Figure 8. Plot of $\lambda$ vs $k$ for Different Values of Dunder's Parameter $\alpha$, for Bi-Material Case

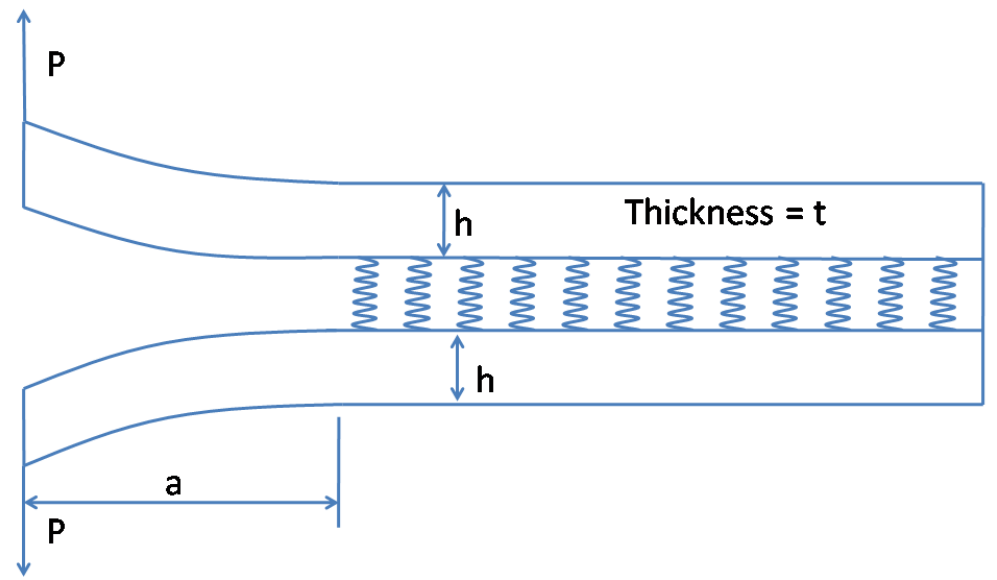

Figure 9. Double Cantilever Beam With Cohesive Zone 


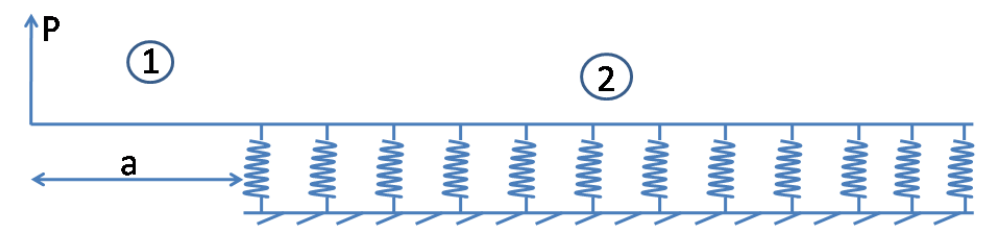

Figure 10. Analytical Model of Double Cantilever Beam

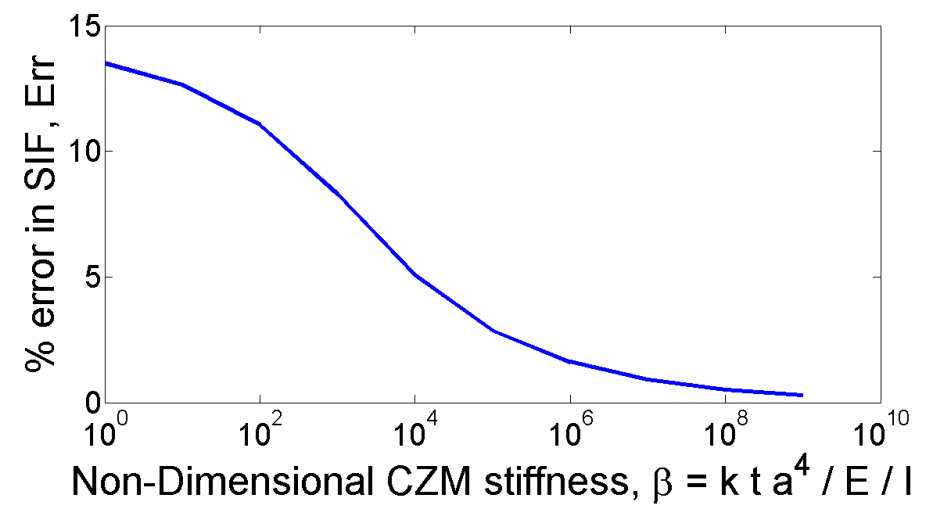

Figure 11. Plot of Percentage Error in SIF of DCB With and Without Cohesive Zone vs. Stiffness of Cohesive Zone 


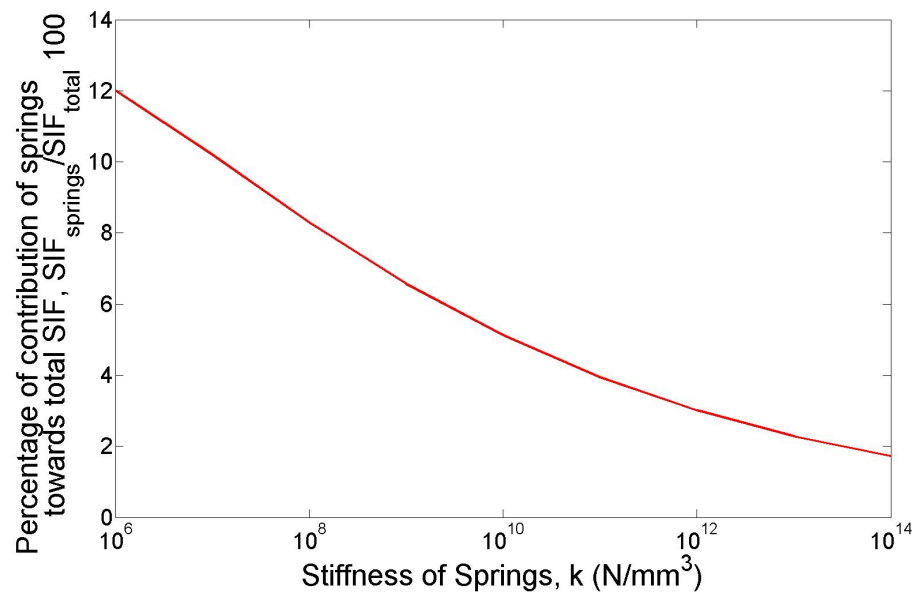

Figure 12. Plot of Percentage Contribution of Cohesive Zone towards SIF of DCB as Compared to the Total SIF of the DCB

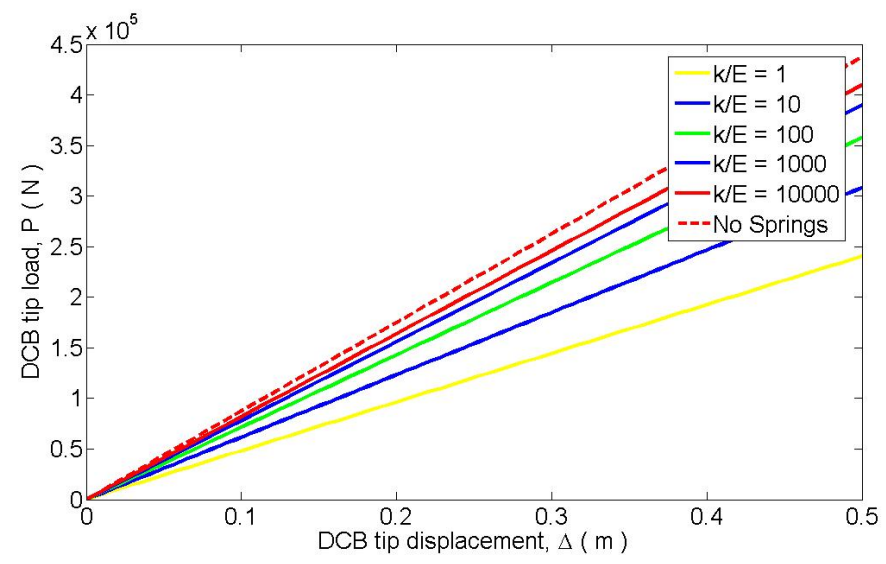

Figure 13. Plot of DCB Tip Load vs Tip Deflection for a Range of Spring Stiffness 


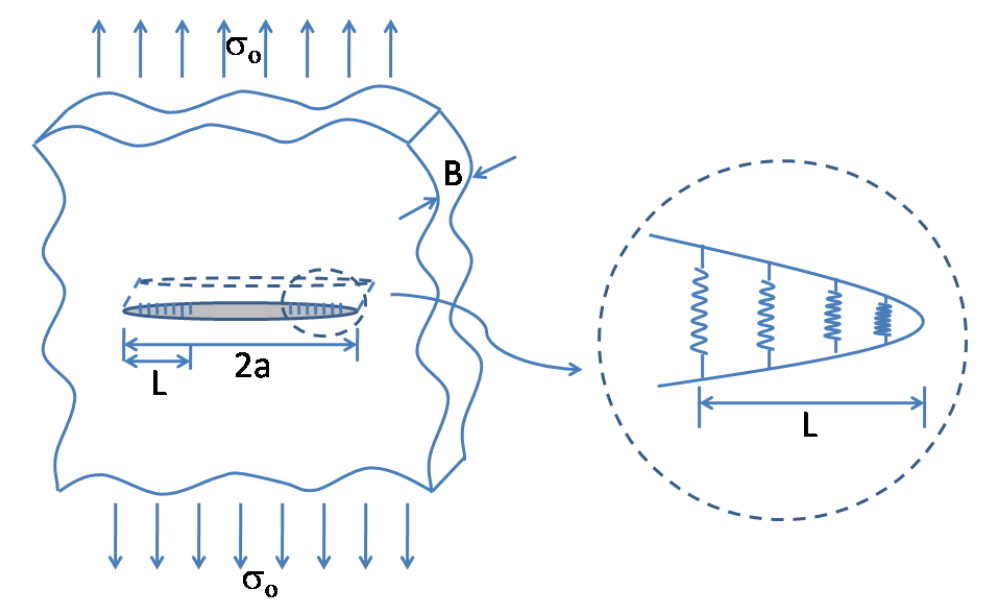

Figure 14. Model of Infinitely Wide Plate With Through Crack Subjected to Tensile Stresses

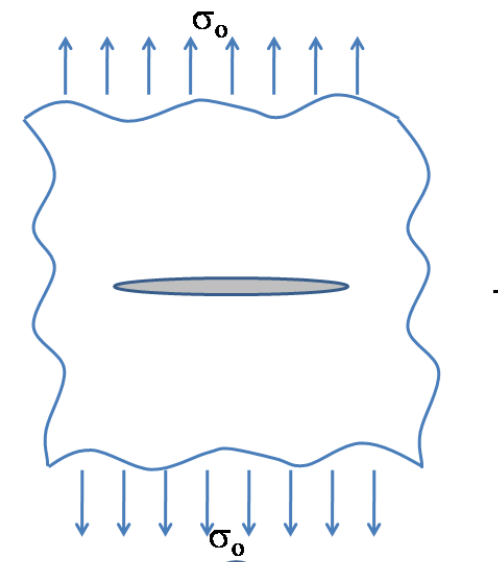

(A)

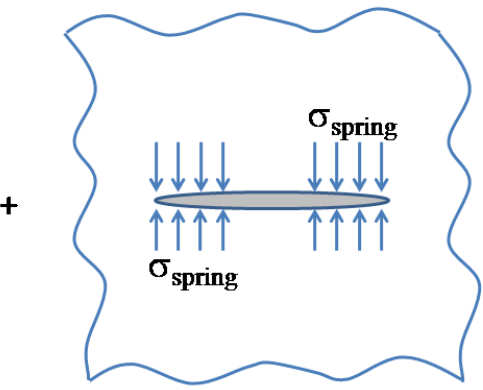

(B)

Figure 15. Superposition to obtain the original Wide Plate with through Crack subjected to Tensile Stresses 


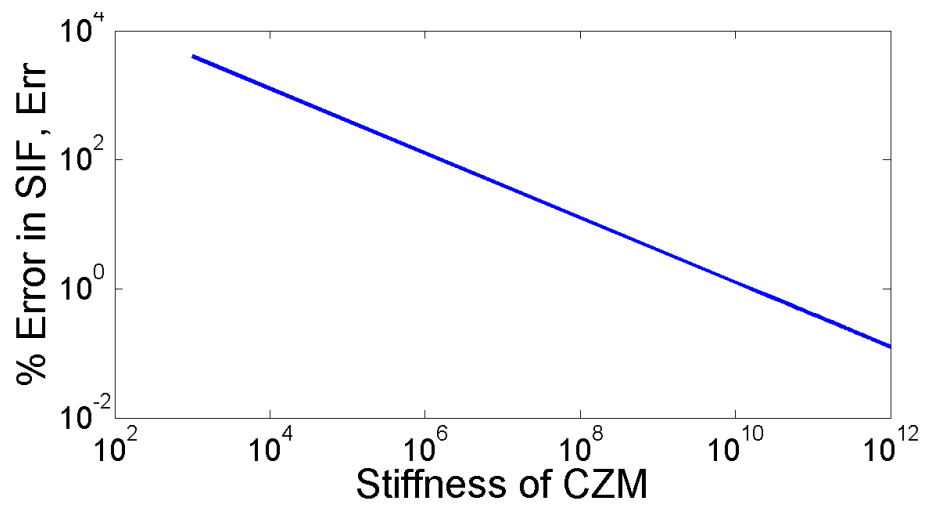

Figure 16. Plot of Percentage Error in SIF of Plate With and Without Cohesive Zone vs. Stiffness of Cohesive Zone, $k$

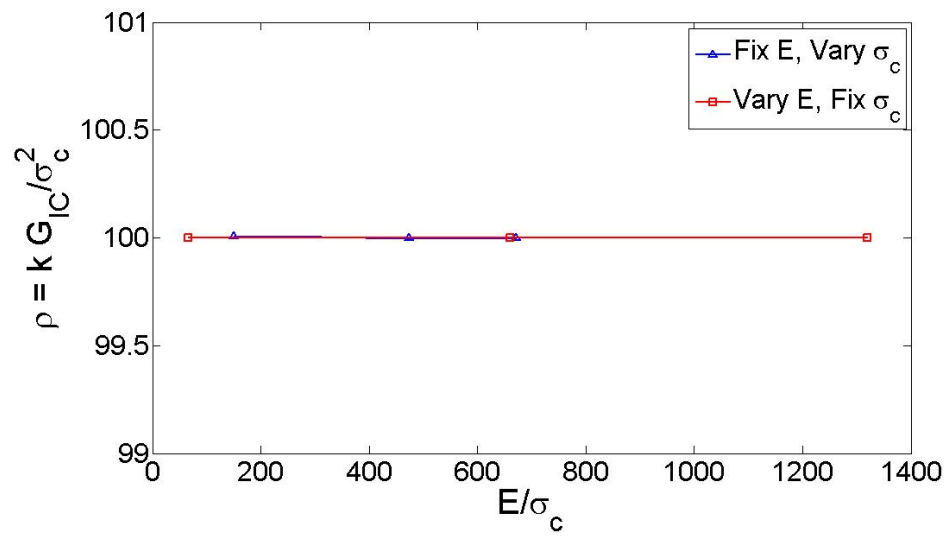

Figure 17. Plot of Non-Dimensional parameters $\rho$ and $E / \sigma_{c}$ for different $L$ and for the Two Cases

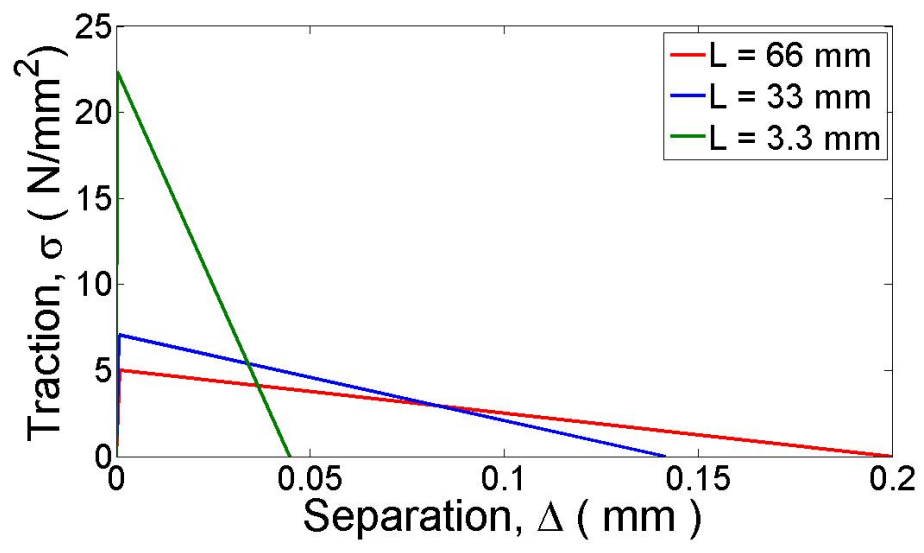

Figure 18. Cohesive Law for $\mathrm{L}=66 \mathrm{~mm}, 33 \mathrm{~mm}$ and $3.3 \mathrm{~mm}$ corresponding to case 1 